\title{
Internal Variability of the Winter Stratosphere. Part II: Time-Dependent Forcing
}

\author{
R. K. SCOTT \\ Northwest Research Associates, Bellevue, Washington \\ L. M. POlvani \\ Department of Applied Physics and Applied Mathematics, Columbia University, New York, New York \\ D. W. WAUGH \\ Department of Earth and Planetary Science, The Johns Hopkins University, Baltimore, Maryland
}

(Manuscript received 11 September 2007, in final form 10 December 2007)

\begin{abstract}
This paper considers the effect of time-dependent lower boundary wave forcing on the internal variability found to appear spontaneously in a stratosphere-only model when the forcing is perfectly steady. While the time-dependent forcing is found to modulate the internal variability, leading in some cases to frequency locking of the upper-stratospheric response to the forcing, the temporal and spatial structure of the variability remains similar to the case when the forcing is time independent. Experiments with a time-periodic modulation of the forcing amplitude indicate that the wave flux through the lower boundary is only partially related to the instantaneous forcing, but is more significantly influenced by the condition of the polar vortex itself. In cases of purely random wave forcing with zero time mean, the stratospheric response is similar to that obtained with steady forcing of magnitude equal to the root-mean-square of the time-varying forcing.
\end{abstract}

\section{Introduction}

In a companion paper (Scott and Polvani 2006, hereafter Part I) it was demonstrated that a realistic stratosphere (considered in isolation) possesses its own natural or internal variability, in the sense that, in the absence of any time dependence in the external forcing, the stratospheric flow evolves into a time-dependent regime consisting of quasi-periodic vacillations resembling stratospheric sudden warmings. By external forcing, we refer to forcing by processes external to the stratosphere. In the present context, these consist of the excitation of upward-propagating planetary-scale waves from tropospheric sources, as well as radiative forcing of the stratospheric zonal mean flow. In considering purely steady external forcing, we wished to isolate variability that was able to arise from the wavemean flow interactions within the stratosphere itself

Corresponding author address: R. K. Scott, Northwest Research Associates, Inc., P.O. Box 3027, Bellevue, WA 98009-3027.

E-mail:scott@nwra.com from variability that might be imposed externally. Under these artificial conditions, however, the stratospheric variability that resulted was quantitatively similar to observed variability of the stratosphere, consisting of periods of rapid wave-induced vortex deceleration (sudden warmings) separated by periods of more gradual vortex intensification when wave-mean flow interactions were weak and the dominant forcing was through radiative relaxation. Moreover, the variability was found to be robust over a wide range of physical and numerical parameters. Part I therefore demonstrated the robustness of stratospheric variability similar to that observed in many previous studies that used idealized or severely truncated models with few degrees of freedom (e.g., Holton and Mass 1976; Yoden 1987; Christiansen 2000; Scott and Haynes 2000).

The results of Part I raise the question as to the relative importance in the stratosphere of internal variability as compared to variability arising from timedependent external forcing. To address this question, herein we consider the generation of planetary-scale waves from tropospheric sources as an external wave 
forcing for the stratosphere. This "external" wave forcing includes forcing from topography and land-sea temperature contrasts, which might be considered to give rise to a steady component, as well as a part arising from transient synoptic-scale eddies, which gives rise to a component that is variable in time. We have already demonstrated that steady forcing alone is sufficient to generate realistic stratospheric variability, but there is also the possibility that stratospheric variability is generated externally by the time-dependent forcing. There is strong evidence that tropospheric variability can force variability of the stratosphere (e.g., Polvani and Saravanan 2000), in the spirit of Matsuno's (1971) model of the stratospheric sudden warming forced by transient tropospheric wave forcing. At one extreme one could claim that all stratospheric variability arises purely in response to tropospheric variability and that the internal variability described in Part I will be completely dominated by variability arising from time dependence of the forcing. On the other hand, it is increasingly accepted that the state of the stratosphere does indeed play an important role in how effectively waves forced in the troposphere can propagate to higher altitudes (e.g., Waugh and Dritschel 1999; Polvani and Waugh 2004; Esler and Scott 2005). At the other extreme, therefore, one might claim that tropospheric transience is only of secondary importance in determining the stratospheric variability.

The present paper is a first step toward answering the question of to what extent the stratospheric variability can be considered a response to variability arising in the troposphere, and to what extent it can be considered a result of internal stratospheric dynamics of the form described in Part I. One caveat is that, in reality, forcing of the stratosphere is never truly external. In other words, we cannot strictly consider the stratosphere in isolation from the rest of the atmosphere, with independently prescribed forcing. The stratosphere is an integral part of the whole atmosphere and the coupling between it and other regions is nonlocal and nontrivial. Addressing this issue will ultimately require the careful use and analysis of coupled troposphere-stratospheremesosphere models (e.g., Taguchi et al. 2001; Polvani and Kushner 2002). A related but separate issue concerns the response to variability of the radiative forcing in the form the seasonal cycle (e.g., Kushner and Polvani 2006). For example, Scott and Haynes (2002) showed variations in the strength of upward wave propagation that were correlated with variations of the polar vortex resulting from the seasonal cycle. Nonetheless, aspects of the question can still be addressed within the context of a simple stratosphere-only model, and in this first step we retain the concept of external forcing.

Following Part I, we therefore adopt a simplified modeling approach to assess specifically the extent to which the internal stratospheric variability obtained under steady forcing retains its character when the forcing becomes time dependent. Remaining within the framework of the stratosphere-only model, subject to the caveats discussed above and in Part I, we have the advantage of having complete control over the time dependence of the wave forcing, which takes the form of a prescribed lower boundary condition. Such forcing can be considered as perhaps the simplest representation of tropospheric variability. Subsequent work will attempt the considerably more challenging problem of extending the current methodology to the context of the coupled troposphere-stratosphere system.

The paper is organized as follows: In section 2, we briefly describe the model and the form of the time dependence of the forcing used in the study. In section 3 , we present results for the case in which the steady component of the forcing is modulated by a periodic component with frequency and amplitude as control parameters. In section 4, to more closely match the observed frequency distribution of tropospheric wave amplitudes, we extend the analysis to forcing modulations that contain a full spectrum of frequencies. In section 4 we also consider a variation, in which the steady forcing is replaced by a time-varying signal with zero time average, that is, the steady component of the forcing is exactly zero. In section 5 we present our conclusions.

\section{Time modulation of wave forcing}

We consider a stratosphere-only system with prescribed lower boundary wave forcing and radiative relaxation to perpetual winter thermal structure. The numerical model uses the pseudospectral method, and all numerical details are as described in Part I. Here, we summarize the form of the forcing and list the particular choice of physical parameters used for a control simulation.

Radiative forcing is here specified exactly as in Part I, with a Newtonian relaxation (at uniform time scale $\tau=$ 10 days) to a height-dependent cold anomaly over the winter pole [Part I, Eqs. (1)-(3)]. The strength of the cooling is controlled by a single parameter $\gamma$, and in all of the simulations below we consider the single value $\gamma=1$.

Waves are forced by specifying the geopotential height at the lower boundary. Again, the wave forcing is as specified in Part I, but now includes a simple time 
dependence. Specifically, we replace Eq. (5) in Part I for the geopotential height perturbation $\Phi_{s}^{\prime}$ with

$$
\Phi_{s}^{\prime}=g h(t) G(\phi) \cos (m \theta)
$$

where $h(t)$ represents the time-varying forcing amplitude, specified below. The function $G(\phi)$ is a broad function of latitude $\phi, g$ is gravity, $\theta$ is longitude, and $m$ is the zonal wavenumber (see Part I for full details). Note that the wave forcing is stationary in longitude. In all simulations below we restrict attention to the $m=2$ case, but note that Part I found similar internal variability for the $m=1$ case. One caveat is that this simple configuration excludes traveling waves, which would be expected to play an additional role in determining the stratospheric response depending on their phase speeds relative to the polar vortex (Esler and Scott 2005). This artificial simplification is justifiable in a first analysis, but we note that a complete treatment will ultimately require consideration of the dependence of the variability on traveling waves.

The time dependence of the forcing is specified as a combination of steady forcing plus a time-dependent modulation:

$$
h(t)=h_{0}[1+f(t)] .
$$

The steady forcing case considered in Part I is therefore recovered for $f(t) \equiv 0$. In all of the simulations below, with the exception of those presented in section $4 \mathrm{~b}$, we use the value of $h_{0}=600 \mathrm{~m}$, representing the height in meters of the amplitude of the steady forcing. Note that the forcing amplitude $h(t)$ refers to the multiplier of cos $m \theta$, rather than the rms wave amplitude over longitude. Negative values of $h$ can occur, therefore, and correspond simply to a phase reversal.

As described in Part I, the above choice of forcing parameters, namely, $h_{0}=600, m=2$, and $\gamma=1$, leads to regular internal stratospheric variability in the form of quasi-periodic vacillations, with a period of approximately 60 days, in the steady forcing case. Furthermore, the variability obtained with these parameters is representative of a wide range of forcing values. This choice therefore constitutes our control simulation, which will be compared against all of the simulations below. For reference and completeness, the variability of the zonal mean zonal velocity $\bar{u}$ at $60^{\circ}$ is shown in Fig. 1; the dominant 60-day period of the vacillations was estimated from the frequency spectrum of the time series of $u_{j}, \bar{u}$ evaluated at $60^{\circ}$ and $z=41 \mathrm{~km}$, (see Fig. 8 in Part I). The vertical structure over a restricted time interval (for visual clarity) is shown in Fig. 1b. For a detailed description of the dynamical evolution of the vortex with these parameters, see Part I's Figs. 4-7.

In the simulations presented below, we repeat the
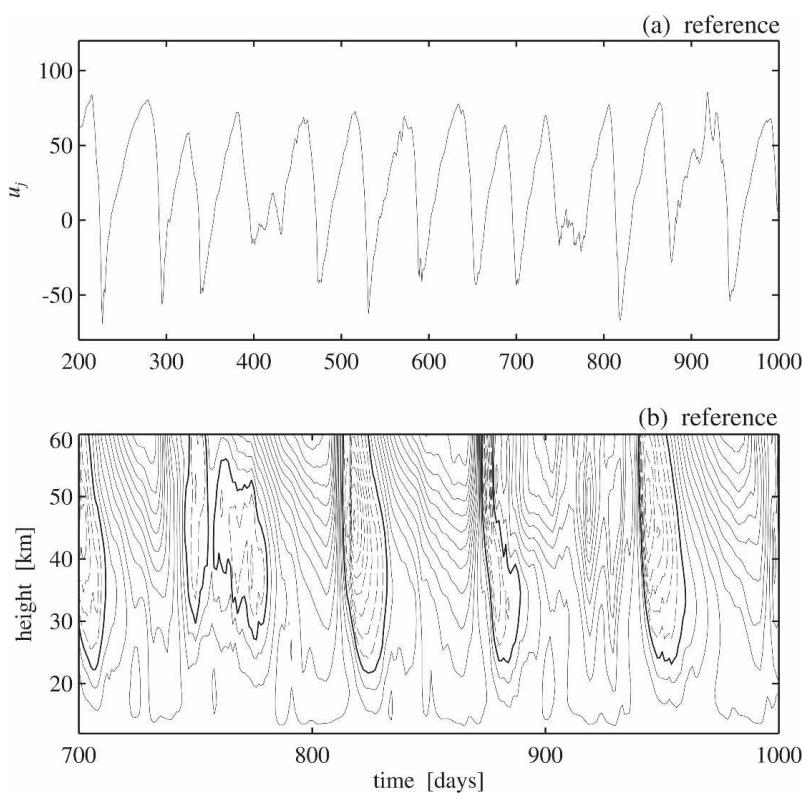

FIG. 1. (a) Zonal mean zonal velocity $\bar{u}$ at $\phi=60^{\circ}$ and $z=41$ $\mathrm{km}$, denoted $u_{j}$, for the reference case with time-independent forcing $h_{0}=600 \mathrm{~m}$ and $\gamma=1$. (b) Corresponding time-height structure over a restricted time interval. The time variability of the response here arises spontaneously. See also Fig. 2b in Part I and related text for further details of the reference case.

calculation leading to Fig. 1 with two different types of forcing modulation. In the first case (see section 3 ), $f$ is a simple periodic function with specified amplitude and period (which we vary as external parameters). In the second case (see section 4), $f$ is a random function with characteristics loosely based on the observed 150-hPa geopotential amplitudes.

\section{Periodic forcing}

We first consider a forcing modulation at a single specific frequency to assess its effect on the internal variability. We add a periodic modulation to the steady forcing to obtain the time-dependent forcing amplitude

$$
h(t)=h_{0}\left(1+a_{f} \sin \frac{2 \pi t}{T_{f}}\right),
$$

where $T_{f}$ is the period and $a_{f}$ is the amplitude of the modulation. We consider forcing periods of $T_{f}=20,40$, 60, 80, and 100 days, and modulation amplitudes $a_{f}=$ $0.1,0.2$, and 0.4 . The largest modulation amplitude of $a_{f}=0.4$ gives a forcing amplitude in the range of $600 \pm$ $240 \mathrm{~m}$ (with a variance of 0.8 times the steady forcing amplitude $\left.h_{0}\right)$.

This form of external forcing time dependence is not intended as an accurate representation of the natural 
variability of tropospheric wave forcing, but rather allows us to address some specific questions concerning the effect of the forcing time dependence on the stratospheric response. By restricting our attention to such simple time-dependent forcing we can determine, for example, which forcing frequencies are effective at generating a response that differs significantly from the internal variability, or the effect on the variability of external forcing frequencies that are close to or far from the natural frequency of the variability, which in this case has a period of 60 days. We can also determine which forcing frequencies, if any, lead to a response that is directly slaved to the forcing period so that the internal variability becomes irrelevant.

Figure 2 shows how the stratospheric response in terms of the zonal mean zonal wind at $60^{\circ} \mathrm{N}$ and $z=41$ $\mathrm{km}$ depends on the modulation period $T$, between 20 and 100 days, for the case of a strong forcing modulation $a=0.4$ (i.e., peak-to-peak variations in the forcing are the same order as the time-average forcing). This should be compared with the response to steady forcing shown in Fig. 1.

Consider first the case of $T_{f}=20$. The most striking feature here is that there is almost no response in the 20-day period. In fact, the upper-stratospheric response consists almost entirely of 60-day periods and closely resembles the internal variability shown in Fig. 1 (this is made quantitative in Fig. 4 below). In other words, the response is apparently independent of the forcing time dependence in this case. We emphasize that the modulation amplitude in this case is large, giving rise to rapid, order of one changes in the forcing amplitude.

At longer modulation periods, in particular for $T_{f}=$ 40 and $T_{f}=80$, the stratospheric variability appears to become frequency locked to the forcing: the dominant periods in the response of these two cases are 40 and 80 days, respectively, although the frequency locking is not perfect and power exists at other periods (again see Fig. 4 below). At the same time, however, the stratospheric response retains the main characteristics of the internal variability, namely, a gradual growth of the vortex followed by a rapid deceleration. Note that for $T_{f}=60$, there is less evidence of frequency locking. This is perhaps surprising, because the forcing frequency in this case almost exactly matches the frequency of the internal variability.

For the case $T_{f}=100$, we find a regime-like response, characterized by long intervals between $t=200$ and $t=$ 300 and between $t=500$ and $t=700$, where the vortex remains persistently strong. The strong vortex regimes are separated by regimes that resemble the internal variability more closely. Longer time series and/or mul-
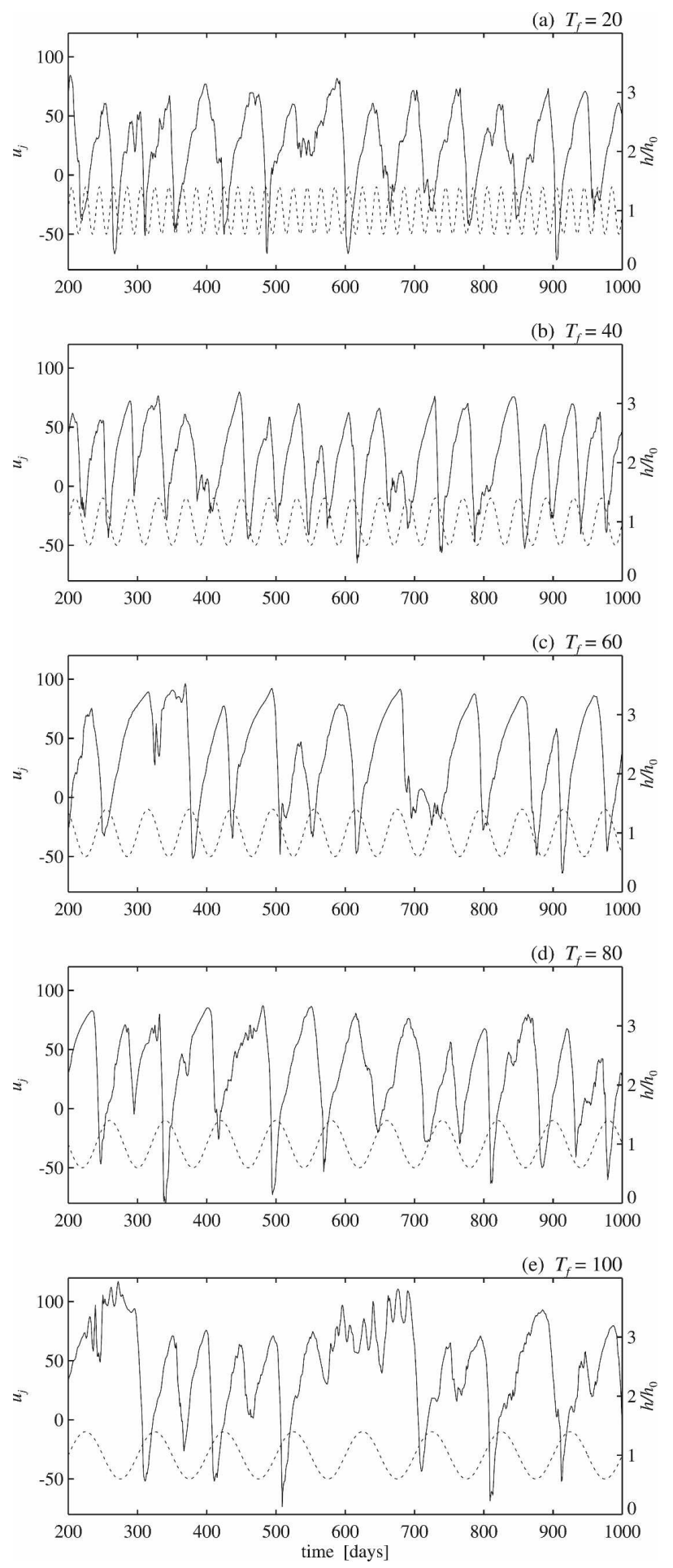

FIG. 2. Zonal mean zonal velocity $\bar{u}$ at $\phi=60^{\circ}$ and $z=41 \mathrm{~km}$ for the case of a purely periodic forcing modulation with period $T_{f}$ and modulation amplitude $a_{f}=0.4$ : (a)-(e) $T_{f}=20$ to $T_{f}=100$ days. Dotted line indicates the forcing function $h(t)$. 
tiple realizations would be required to determine whether the regime-like behavior is robust, but the recurrence of the internal variability is clear over the time interval considered.

The vertical structure of the stratospheric response is shown in Fig. 3. Again, there is a broad similarity in all cases with the vertical structure of the reference case (Fig. 1b). The response is characterized in all cases by a gradual intensification followed by a more rapid deceleration, which often (though not always) begins first at the upper levels and migrates downward. Note again the absence of coherent variability near the forcing period in the case of $T_{f}=20$ days.

The dependence on $a_{f}$ is less marked. At smaller $a_{f}$ (not shown) the variability is also largely unaltered in character (i.e., the response closely resembles the internal variability) and there is very little systematic variation with $T_{f}$ at small $a_{f}$. To summarize the behavior across the full range of parameters, in Fig. 4 we show the frequency power spectra of the stratospheric response, defined as $u_{j}(t)=\bar{u}\left(\phi=60^{\circ}, z=41 \mathrm{~km}\right)$, over the time interval $200 \leq t \leq 1000$ days for each forcing period and modulation amplitude. The spectra at other levels show similar characteristics. This makes the statement above for the $T_{f}=20$ case (left-hand column) - that the response contains very little power at the forcing frequency (dashed lines)-more quantitative; rather, the response is dominated by the frequency of the internal variability (heavy gray line). This also can be seen to be true at the smaller forcing modulation amplitudes considered. In these cases we conjecture that the vortex does not reform quickly enough under the effect of radiative relaxation after a vortex breakdown (sudden warming) to allow for frequency locking with the forcing.

At other forcing periods $T_{f} \geq 40$ frequency locking occurs provided that the amplitude is large enough; typically $a_{f} \geq 0.4$, but for $T_{f}=80$ also at $a_{f}=0.2$ (cases in Fig. 4 are marked with a symbol in the upper-lefthand corner). The exception is for $T_{f}=60$, where the forcing frequency matches the internal frequency and for which increasing the amplitude appears to destroy the coherence of the variability by shifting the response to lower frequencies.

In Fig. 5, we show the relationship of the lower boundary wave flux into the stratosphere with the forcing time dependence and the stratospheric response. The wave flux (solid) is defined as the vertical component of the Eliassen-Palm (EP) flux evaluated at the lower boundary, averaged over the Northern Hemisphere, that is, $\int_{0}^{\pi / 2} F^{(z)} \cos \phi d \phi$, where $\mathbf{F}=\left[F^{(\phi)}, F^{(z)}\right]$ is the EP flux as defined by Andrews et al. [1987, their
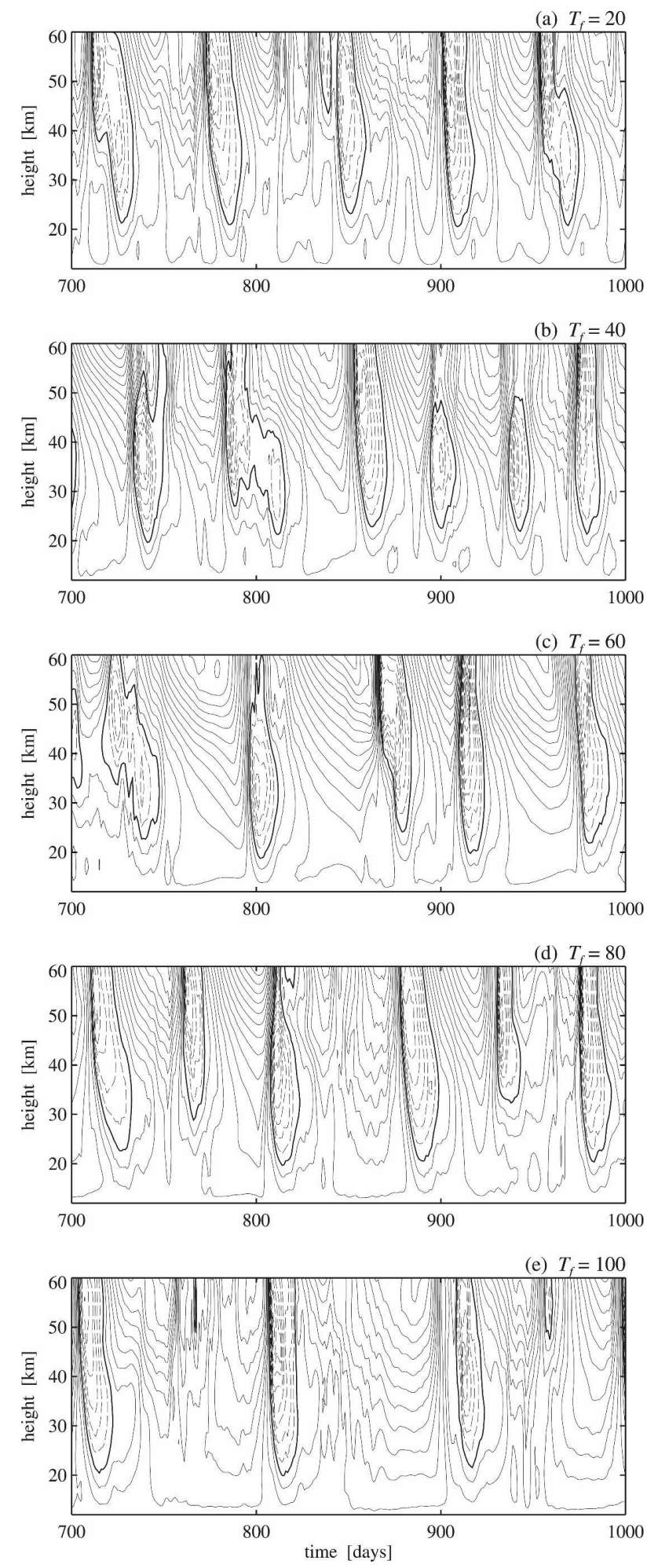

FIG. 3. Zonal mean zonal velocity $\bar{u}$ at $\phi=60^{\circ}$ for the case of a purely periodic forcing modulation with period $T_{f}$ and modulation amplitude $a_{f}=0.4$ : (a)-(e) $T_{f}=20$ to $T_{f}=100$ days. 

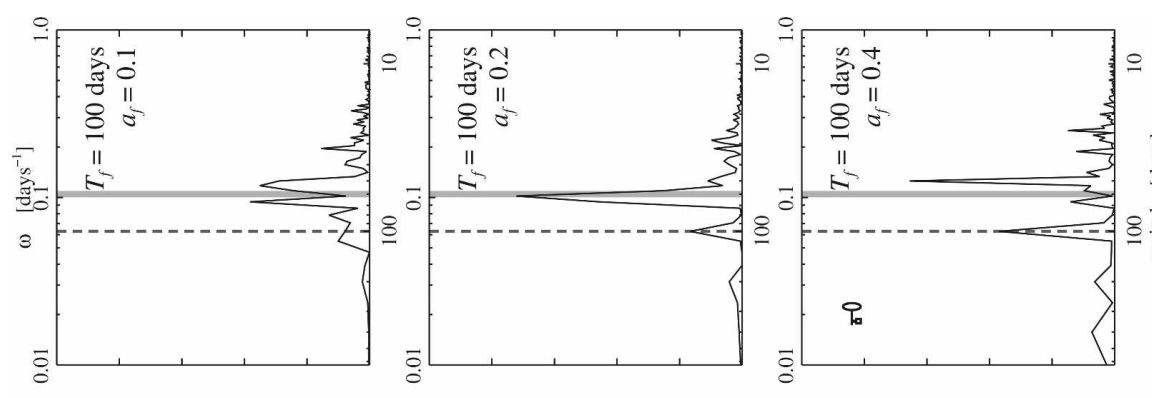

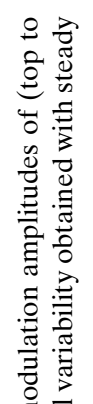
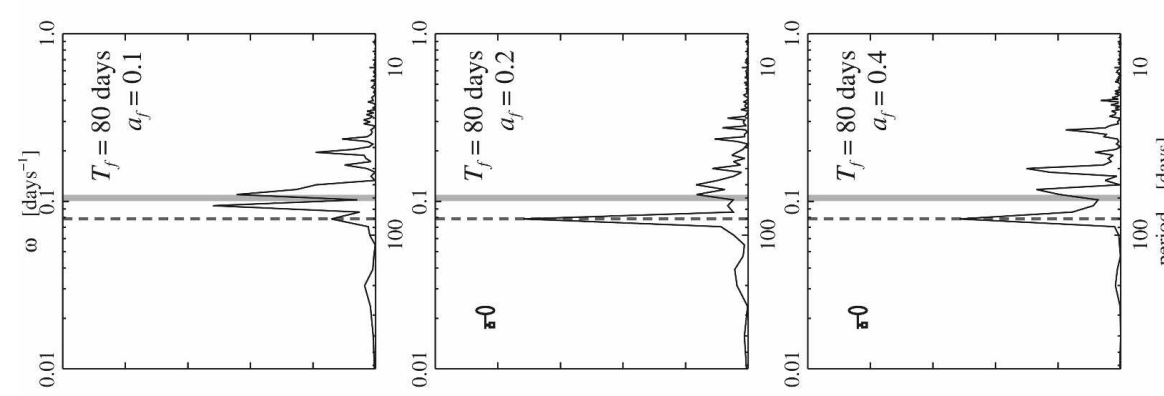

ป
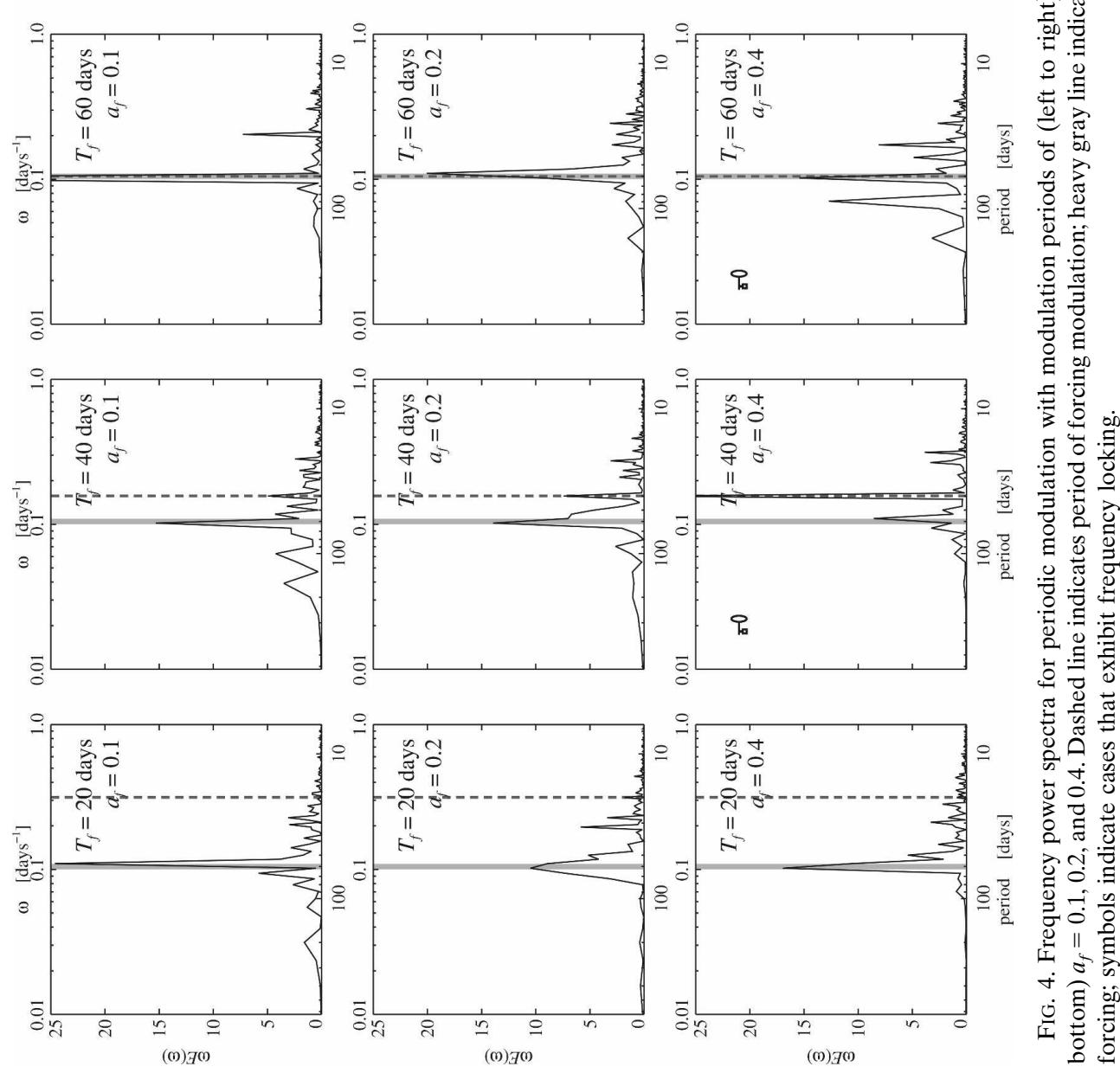
(a) $T=20$

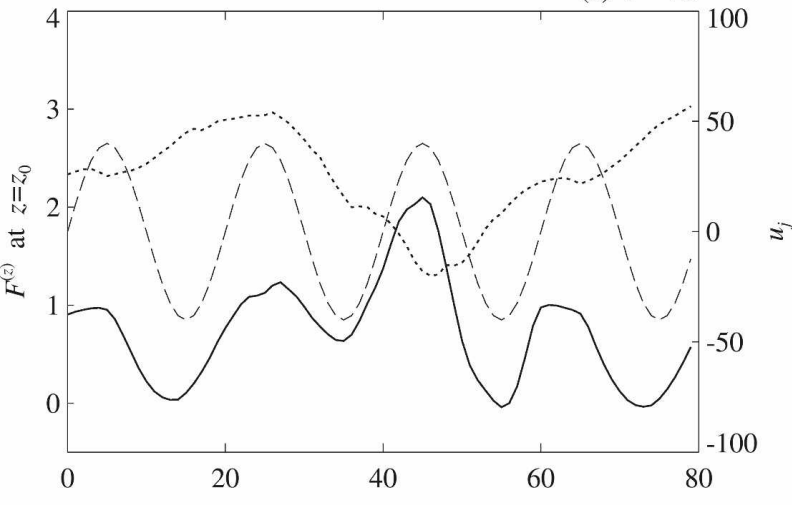

(b) $T=40$

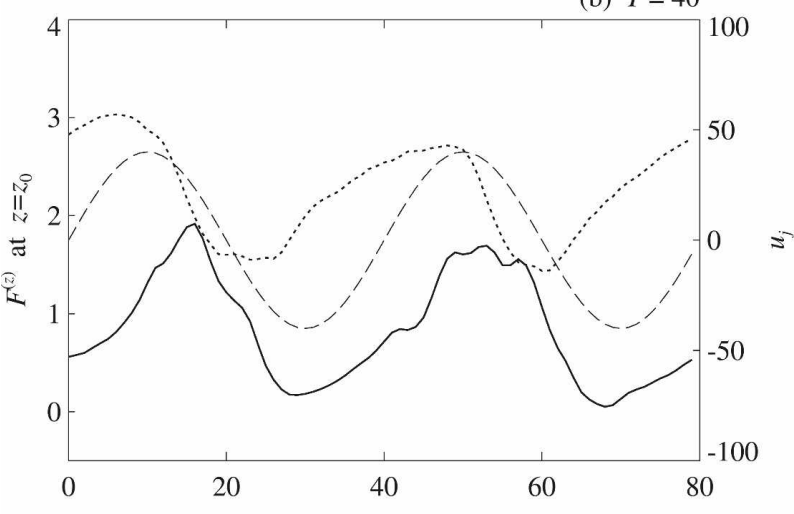

(c) $T=80$

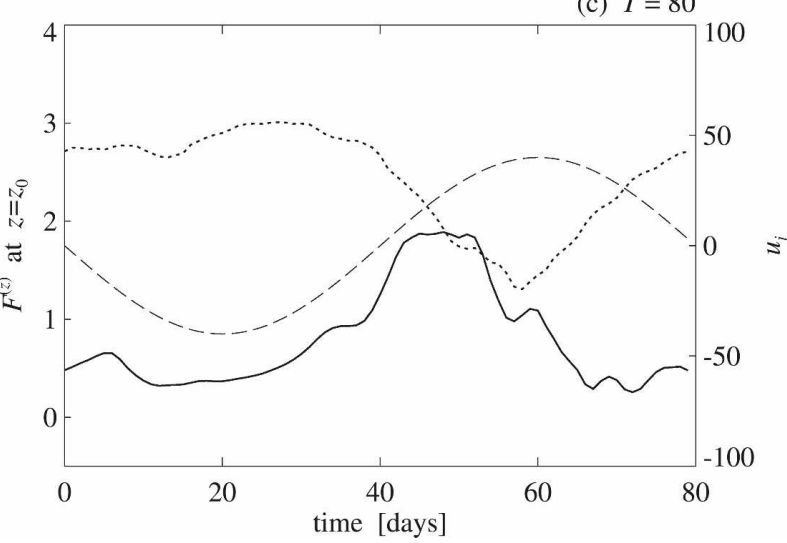

FIG. 5. Composite of the vertical EP flux at the lower boundary $\left.F^{(z)}\right|_{B}$ averaged over latitude (solid) and composite of $\bar{u}$ at $\phi=$ $60^{\circ}$ latitude and $z=41 \mathrm{~km}$ (dotted) for the case of periodic forcing modulation with amplitude $a_{f}=0.4$ and period $T_{f}=$ (a) 20, (b) 40, and (c) 80 days. Composites are taken over consecutive 60-, 80-, and 80-day periods in (a)-(c), respectively. The forcing amplitude is also shown (dashed).

Eq. (3.5.3)]. The stratospheric response (dotted) is defined as $\bar{u}$ at $\phi=60^{\circ}$ latitude and $z=41 \mathrm{~km}$. The key points are illustrated with the $a_{f}=0.4$ and $T_{f}=20,40$, and 80 cases, which also allow for convenient and in- tercomparable composites. We plot composites of both quantities averaged over consecutive 80-day intervals, that is, $t \in[200,280],[280,360], \ldots$, for the $T_{f}=40$ and $T_{f}=80$ cases (where the response is frequency locked to 40- and 80-day cycles, respectively) and averaged over consecutive 60-day intervals for the $T_{f}=20$ case (where the internal period is dominant). In each composite interval, the lower boundary wave forcing (dashed) has the same phase.

Considering first the stratospheric response (dotted), frequency locking with the forcing is clear for the $T_{f}=$ 40 and $T_{f}=80$ cases but is absent for the $T_{f}=20$ case, where the response is at the natural frequency. Similarly, considering the lower boundary EP flux, there is also a tendency for the peaks in this quantity to align with the maximum forcing amplitude. In the $T_{f}=40$ and $T_{f}=80$ cases, the EP flux begins to increase roughly at the same time as the forcing amplitude, that is, when the forcing amplitude is at a minimum. The time taken for the EP flux to peak, however, appears to be independent of the forcing period, so that at $T_{f}=80$ the EP flux peaks before the maximum forcing amplitude, whereas at $T_{f}=40$ the EP flux peaks after the maximum. Thus, the relation between the wave forcing and the EP flux is not one to one, but rather the state of the stratosphere is crucial for determining what EP flux results from a given forcing. This point has been made previously for the case of steady forcing (Scott and Polvani 2004), as well as for the case of transient (traveling) wave forcing (Esler and Scott 2005).

Even for the $T_{f}=20$ case in which no frequency locking occurs, there is clear alignment of peaks in EP flux with the forcing, although only every third forcing maximum corresponds to a strong peak and vortex deceleration. Note that although there is significant highfrequency variability in the EP flux at $T_{f}=20$, the upper-stratospheric response is dominated instead by vacillations with a similar period (60 days) to the internal variability obtained with steady forcing. In this case, the internal variability can be said to survive the addition of strong variability in the wave forcing.

In summary, we find that time dependence in the forcing can influence the timing of the stratospheric response, but in other respects (e.g., the amplitude and structure of the response) it does not appear to have a strong effect. Forcing frequencies close to that of the internal variability can alter the period of the stratospheric response through frequency locking. On the other hand, higher forcing frequencies cannot project onto the internal variability because the time scale for vortex recovery exceeds the forcing time scale. Also, for small modulation amplitudes no frequency locking is obtained. Even in cases where frequency locking oc- 
curs the stratospheric response is not otherwise linked to the time dependence of the forcing, in the sense that the other characteristics of the response (gradual vortex intensification followed by rapid deceleration) closely resemble the internal variability. The spatial structure of the vortex buildup and breakdown events (see Fig. 8 below) is also found to be very similar to that of the time-independent forcing case, regardless of the forcing period or amplitude.

\section{Aperiodic forcing}

Periodic forcing is useful for examining the response to particular forcing frequencies, but is much too simple to be a good approximation of the observed tropospheric variability. Therefore, we now examine how the nature of the stratospheric response changes when the wave forcing more closely resembles observations, which contain a broad range of frequencies. To this end we consider the time dependence of the observed geopotential height amplitude near the tropopause. Figure 6 shows the time series and frequency power spectrum for the wavenumber two geopotential height amplitudes at $60^{\circ} \mathrm{N}$ and $p=150 \mathrm{hPa}$ derived from the 19792006 National Centers for Environmental Prediction (NCEP) reanalysis. The time series is obtained from five (typical) winter seasons (December-April) for the Northern Hemisphere, concatenated into a single interval. Note that there are many different time scales evident in the time series. Note also that on average (lower panel) the power is broadly uniform across the frequency range of $0.06-0.3$ days $^{-1}$, corresponding to periods of 100-30 days $(\omega=2 \pi / T)$, while at higher frequencies the power falls off gradually. At still lower frequencies (not shown) the power remains approximately uniform apart from strong peaks at the annual cycle and its harmonics.

The random forcing modulation that we use in this section is based loosely on the frequency spectrum shown in Fig. 6. It consists of a random superposition of frequencies in the range of $2 \pi / T_{\max }-2 \pi / T_{\min }$, where $T_{\max }=1000$ days is the duration of the simulation and $T_{\min }$ is a high-frequency cutoff. We are not concerned here with reproducing the exact frequency distribution, but simply seek a crude approximation with a range of different forcing frequencies. Specifically, we take the wave forcing amplitude to be of the form

$$
h(t)=h_{0}\left[1+a_{f} \sigma(t)\right]
$$

where

$$
\sigma(t)=\sum_{n=1}^{N}\left[\alpha_{n} \sin \left(\omega_{n} t\right)+\beta_{n} \cos \left(\omega_{n} t\right)\right],
$$

(a)

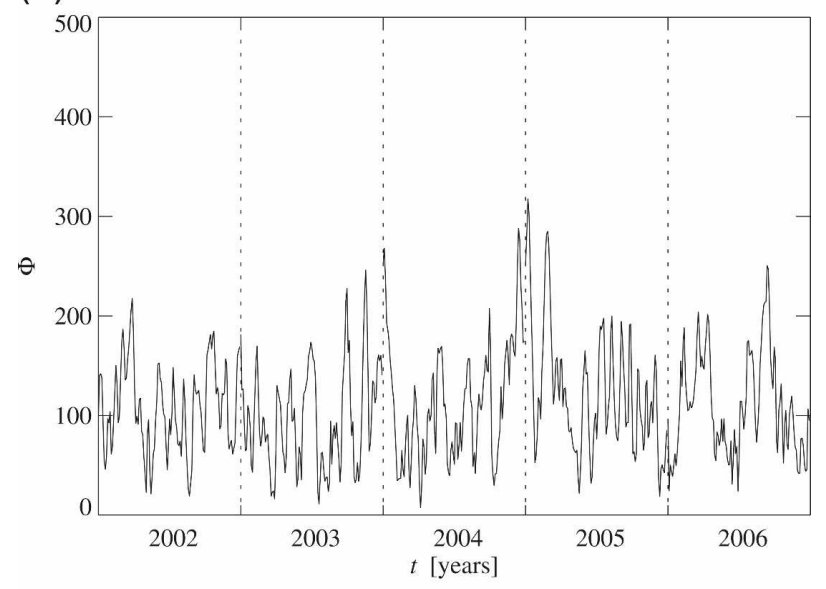

(b)

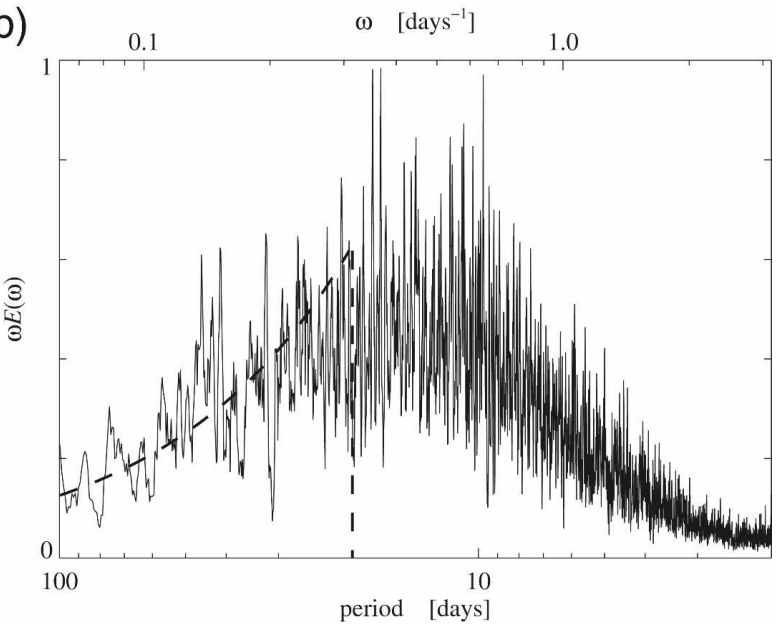

FIG. 6. (a) Time series of wavenumber two geopotential amplitude at $60^{\circ} \mathrm{N}$ and $p=150 \mathrm{hPa}$ for five consecutive winters (defined as 1 Dec-31 Mar). (b) Frequency power spectrum of wavenumber two geopotential amplitude for the full period of 1979 2006 (combines NH and SH; data available online at http:// hyperion.gsfc.nasa.gov/Data_services). The bold dashed line shows schematically the frequency distribution of (5) for the case $T_{\text {min }}=20$.

and where $N=T_{\max } / T_{\min }$ is the number of discrete frequencies, $\omega_{n}=2 \pi n / T_{\max }$, and $\alpha_{n}, \beta_{n}$ are uniformly distributed random variables on $[-1,1]$, satisfying $\alpha_{T}^{2}+$ $\beta_{T}^{2}=1$. With this choice, the power is distributed uniformly across frequencies in the given range. The variance of $\sigma$ is $\operatorname{var}(\sigma)=N / 2$, so that $\operatorname{var}(h)=\langle(h-$ $\left.\left.h_{0}\right)^{2}\right\rangle=a_{f}^{2} h_{0}^{2} N / 2$. We choose $T_{\min }=20$ days for most of the simulations presented in this section, but $T_{\min }=10$ and $T_{\min }=40$ are also used to assess the sensitivity to this parameter. The actual time dependence of the forcing in the simulations presented next are shown in the corresponding figures. 


\section{a. Modulation of steady forcing}

First, we consider a modulation of the steady forcing as just described and given by (4) with $T_{\min }=20$. This low-frequency cutoff is partly justified by the fact that, based on for example, Fig. 5, forcing periods less than $T_{f}=20$ do not project strongly onto the variability. Further, the power spectrum of the observed geopotential in the lower stratosphere begins to fall off at around this frequency (Fig. 6b).

Figure 7 shows the dependence of the variability on the forcing modulation amplitude for $a_{f}=0.02, a_{f}=$ 0.06 , and $a_{f}=0.10$. Recall that $\operatorname{var}[\sigma(t)]=N / 2=25$ for $T_{\min }=20$ so that the standard deviation of the forcing at $a_{f}=0.1$ is $\sqrt{\operatorname{var}(h)}=0.5 h_{0}$, that is, half of the steady forcing amplitude. The dotted lines indicate the actual forcing time dependence. At all modulation amplitudes (as well as at intermediate amplitudes that are also considered, but not shown here) the dominant response is at the period of the internal variability, namely, 60 days. This has been verified by considering the frequency power spectra, as in Fig. 4 above (but not shown here): in all cases there is a distinct peak in the power spectrum at 60 days, although there is also more spread among other frequencies than previously, indicating some increase in low-frequency variability.

A natural question is whether the timing of the sudden warming events can be correlated with the instantaneous amplitude of the forcing. In fact, it was found that even at the largest forcing modulation, the response is not well correlated with the forcing time dependence. For example, the strongest sudden warming in the $a_{f}=0.1$ case (Fig. 7) occurs near $t=480, t=600$, and $t=860$, where there is no clear maximum in forcing amplitude or its time dependence (see dotted line). Conversely, the strong time dependence of $h(t)$ near $t=$ 760 does not result in a strong warming event at that time, simply because it occurs when the vortex happens to be recovering from a previous sudden warming event.

It is interesting to compare the detailed structure of the polar vortex during the sudden warming events between the reference case (steady forcing) and the case with modulated forcing. Figure 8 shows the meridional structure of $\bar{u}$ for composites of all of the warming events for the reference case (top row) and the strongest modulated forcing case, $a_{f}=0.1$. Time $t=0$ of a sudden warming event is defined as the day when $u_{j}>$ $50 \mathrm{~m} \mathrm{~s}^{-1}$ for the preceding 2 days, $u_{j}<50 \mathrm{~m} \mathrm{~s}^{-1}$ for the following 2 days, and $u_{j}<0 \mathrm{~m} \mathrm{~s}^{-1}$ within the following 20 days. There are 13 events in the reference composite and 10 events in the modulated composite. As can be seen from the figure, the structure of the sudden warm-
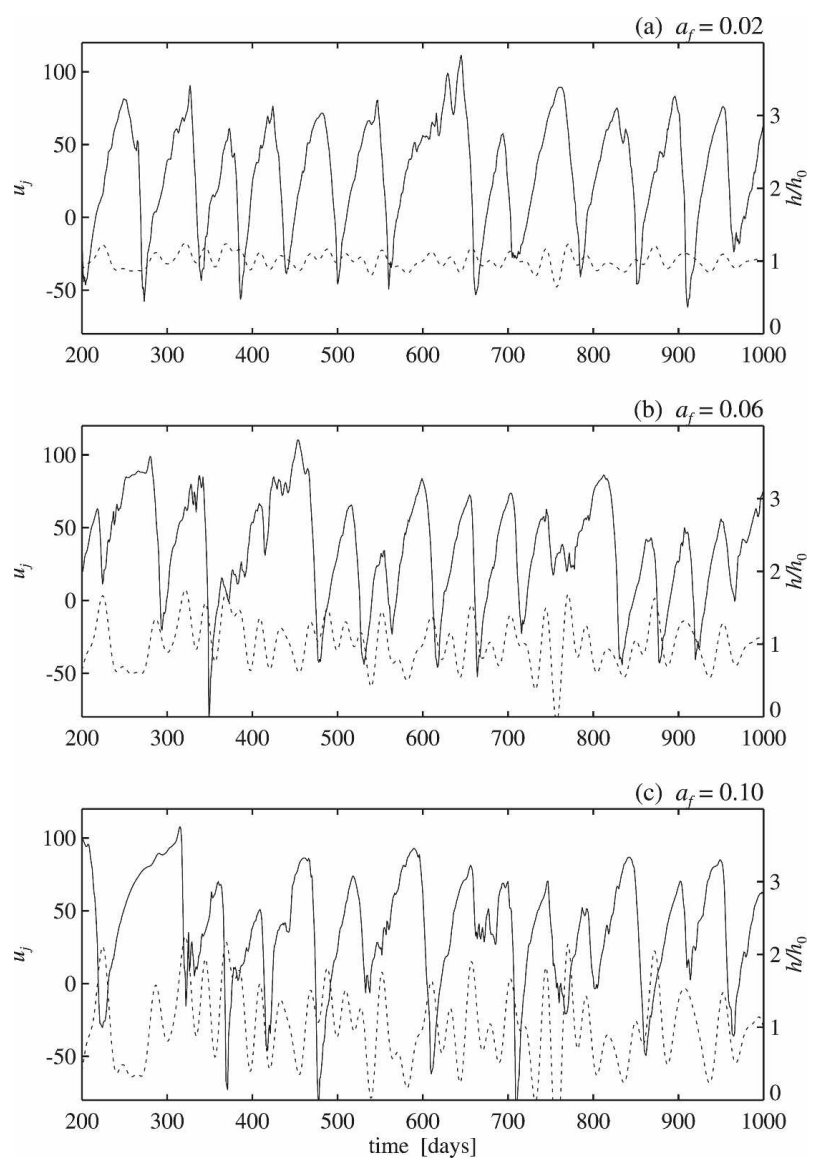

FIG. 7. Zonal mean zonal velocity $\bar{u}$ at $\phi=60^{\circ}$ and $z=41 \mathrm{~km}$, $u_{j}$, for the case of a random forcing modulation: modulation amplitude (a) $a_{f}=0.02$, (b) $a_{f}=0.06$, and (c) $a_{f}=0.10$. Dotted line indicates the forcing function $h(t)$.

ing events between the two cases is very similar. The warming proceeds by a tightening of the polar vortex into high latitudes (left), followed by a rapid deceleration in which the zero wind line moves poleward and westerlies are replaced by easterlies throughout the middle and upper stratosphere. The similarity between the two cases provides further support that the variability found in the case of modulated forcing is determined essentially by the internal variability, rather than by the time dependence of the forcing.

Finally, we consider the dependence of the variability on the high-frequency cutoff. This is shown in Fig. 9 for $T_{\min }=10$ and $T_{\min }=40$. The modulation amplitude has been chosen such that the variance of the forcing is the same in each case; namely, $a_{f}=0.08$ for $T_{\min }=10$ and $a_{f}=0.16$ for $T_{\min }=40$, so that $\sqrt{\operatorname{var}(h)}=a_{f} h_{0}$ $\sqrt{N / 2} \approx 0.57 h_{0}$ (which is comparable to the $a_{f}=0.1$ and $T_{\min }=20$ case, shown in Fig. 7). 

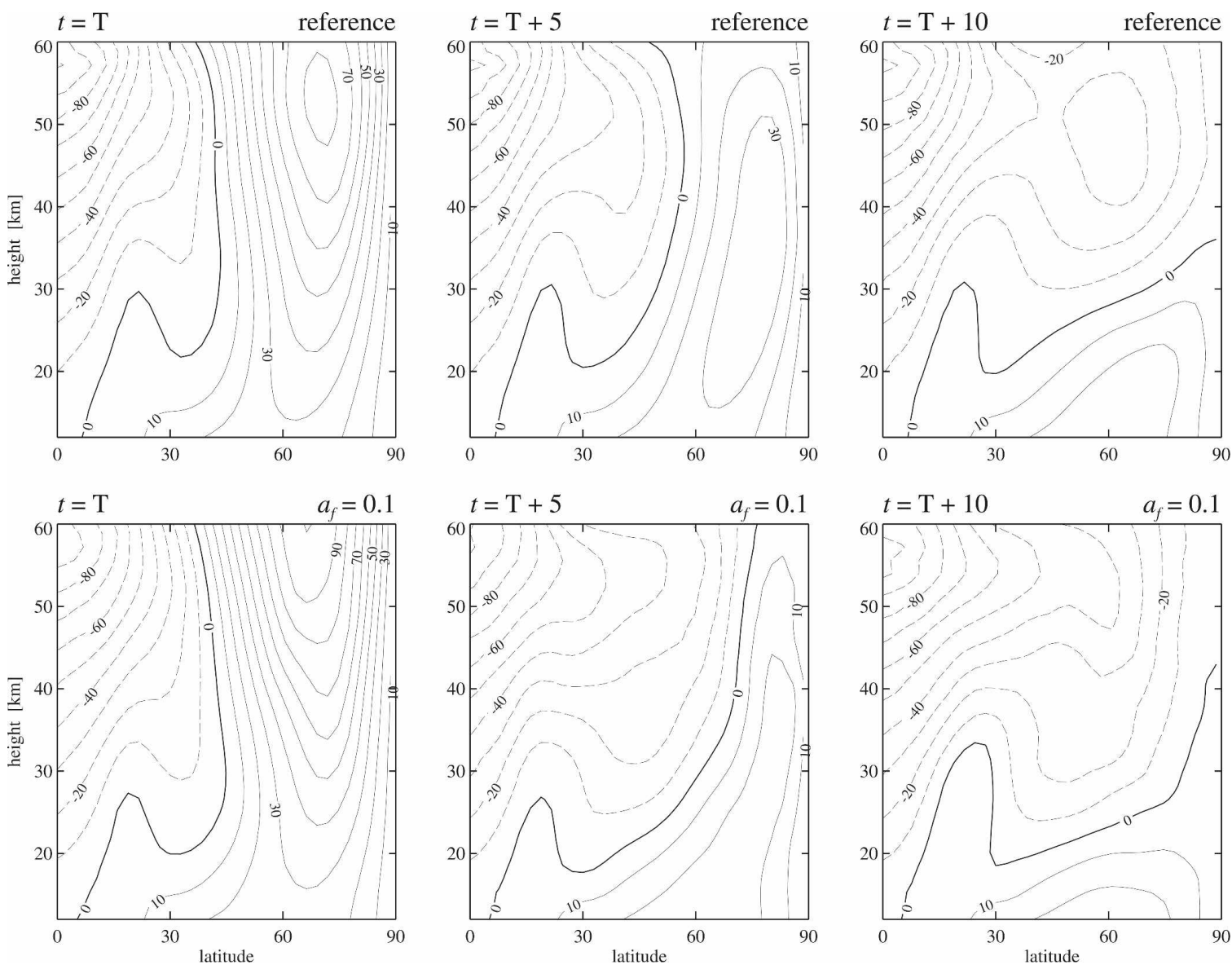

FIG. 8. Composite zonal mean zonal velocity $\bar{u}$ around the onset of (top) 13 sudden warming events from the reference (steady forcing) case and (bottom) 10 sudden warming events from the case with random forcing modulation with $a_{f}=0.1$ and $T_{\min }=20$ days. Time $t=T$ corresponds to the deceleration to below $50 \mathrm{~m} \mathrm{~s}^{-1}$ of $u_{j}=\bar{u}$ at $\phi=60^{\circ}$ and $z=41 \mathrm{~km}$.

For the $T_{\min }=10$ case, we note that most of the high-frequency variability in the forcing projects only to a very limited extent on the stratospheric response. In particular, the dominant stratospheric variability again consists of vacillations at the frequency of the internal variability. Sudden warmings are occasionally aligned with peaks in the forcing amplitude (e.g., $t=220$ and $t=660)$, but on the whole, there is very little correlation. As before, when the forcing peaks occur during an interval of vortex recovery, no sudden warming can occur.

For the $T_{\min }=40$ case, the behavior is very different. Here there are extended intervals where the stratospheric response aligns closely with the forcing amplitude, in particular the intervals of weak forcing between $t=600$ and $t=950$, when the vortex remains relatively undisturbed. On the other hand, there are many sudden warming events that cannot be explained by the forcing amplitude at $t=280, t=440, t=560$, and $t=980$. The stronger correlation in this case arises from the fact that the lower forcing frequencies can project more easily onto the internal stratospheric variability. Again it appears, therefore, that the internal variability can become phase locked with the forcing when the forcing time dependence contains periods of around 40 days.

\section{b. Aperiodic forcing with zero time mean}

In the above, the forcing is represented by a steady component plus a time-dependent modulation. We consider next, as a special case, the situation when the steady component is absent. Specifically, we consider a forcing time dependence with $h(t)$ defined as

$$
h(t)=a_{f} \sigma(t),
$$

with $\sigma(t)$ as before. The amplitude of the forcing is now determined by $a_{f}$, and with $a_{q} \rightarrow a_{q} / h_{0}$ this gives forcing 

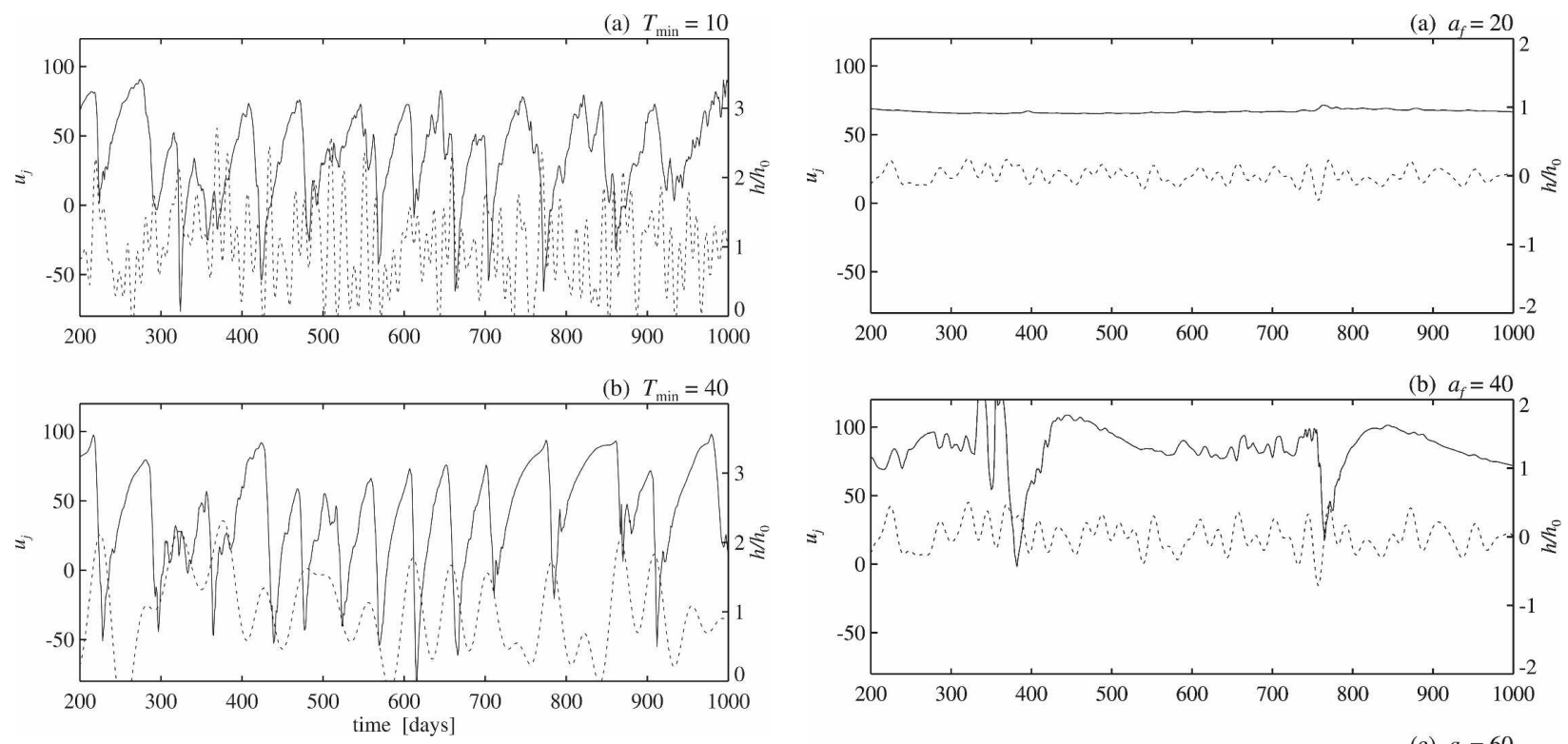

FIG. 9. Zonal mean zonal velocity $\bar{u}$ at $\phi=60^{\circ}$ and $z=41 \mathrm{~km}$, $u_{j}$ (solid) for the case of a random forcing modulation but with different high-frequency cutoff, $T_{\min }$ : (a) $T_{\min }=10$ days and (b) $T_{\min }=40$ days. Dotted line indicates the forcing function $h(t)$. See Fig. $7 \mathrm{c}$ for the corresponding case with $T_{\min }=20$ days.

with the same variance as before. In this case, the most meaningful measure of the forcing is the root-meansquare of the forcing amplitude, which is $h_{\mathrm{rms}}=a_{f}$ $\sqrt{N / 2}=5 a_{f}$, in the case $T_{\min }=20$. For $T_{\min }=20$ days the time dependence of $h$ is as shown in the dotted lines in Fig. 7, but the abscissa is shifted by 1 so that the forcing has zero time mean.

One reason for considering the case of forcing with zero time mean is that steady forcing, if it is greater than a critical value (see, e.g., Fig. 11 from Part I), always gives rise to internal variability. From the results presented above, it appears that this is the case even when the steady forcing is modulated by a time-varying component. In other words, the response to the steady component of the forcing appears to dominate the response to the time-dependent modulation. We may also ask, however, whether a steady component of the forcing is even necessary for exciting the internal variability, and whether the response to forcing with zero time mean will be different from that described thus far.

Figure 10 shows the dependence of the variability on the forcing amplitude $a_{f}$; the response is qualitatively different from before. At $a_{f}=20\left(h_{\mathrm{rms}}=100\right)$, the wave forcing is too weak to have any large impact on the
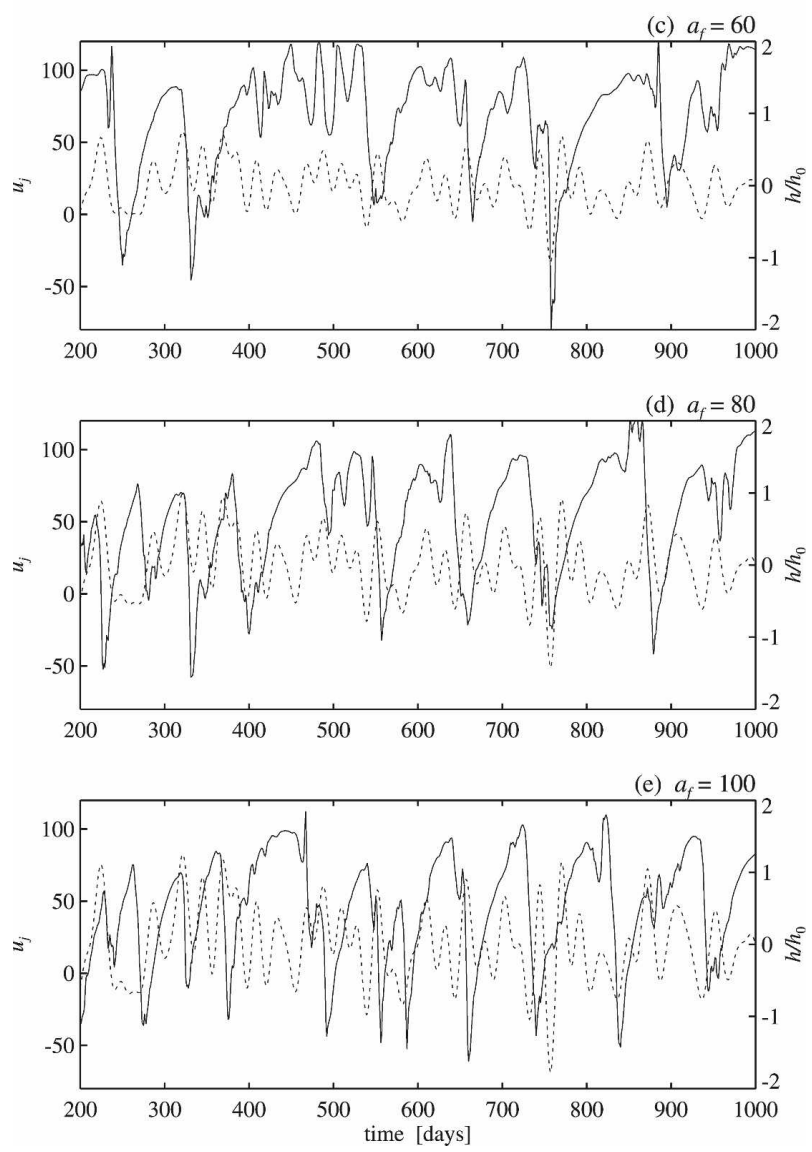

FIG. 10. Zonal mean zonal velocity $\bar{u}$ at $\phi=60^{\circ}$ and $z=41 \mathrm{~km}$, $u_{j}$ (solid), for a random forcing with zero time mean, for forcing amplitudes $a_{f}=$ (a) 20, (b) 40, (c) 60, (d) 80, (e) 100. Dotted line indicates the forcing function $h(t)$. polar vortex, which remains close to thermal equilibrium. Tiny fluctuations are visible, coinciding with times when the forcing amplitude is anomalously large 
(from $t=350$ to 400 and near $t=750$ ). At $a_{f}=40$ $\left(h_{\mathrm{rms}}=200\right)$, these fluctuations become larger sudden warming events, again coinciding with the anomalously large forcing (the time dependence of the forcing is the same in all cases). The wind reversals are less strong than those found with steady forcing. Aside from these two sudden warming events, the vortex again remains relatively undisturbed at other times.

At $a_{f} \geq 60\left(h_{\mathrm{rms}} \geq 300\right)$ the sudden warming events become more frequent and more intense. Moreover, the frequency of the sudden warming events appears to saturate at a level similar to that of the response to steady forcing. At these forcing amplitudes, the correlation between warming events and peaks in the forcing is less obvious, because it can happen that the vortex is recovering from a previous sudden warming and is in a weak state at the time when the forcing increases (e.g., near $t=750$ and $t=870$ in the $a_{f}=100$ case). Thus, the variability at large forcing amplitudes seems to depend on the rms of the forcing amplitude (through the parameter $a_{f}$ ), rather than on details of the forcing time dependence. In a sense, the response again becomes qualitatively similar to the internal variability obtained with steady forcing.

The relation between the response and the forcing amplitude can be quantified by considering the mean, standard deviation, and average extrema or envelope of the time series $\bar{u}(t)$ at $\phi=60^{\circ}$ and $z=40 \mathrm{~km}$, as defined in Part I (p. 2768). Figure 11 shows these quantities as a function of the rms $h(t)$, given by $h_{\mathrm{rms}}=5 a_{f}$. Comparing this with Fig. 11b in Part I, we find that the variability depends on $h_{\mathrm{rms}}$ in a manner that is similar to how the internal variability depends on the steady forcing amplitude $h_{0}$. The dominant frequency increases with $h_{\text {rms }}$, though more slowly than in the case of steady forcing. Also, the mean and standard deviation both vary with $h_{\text {rms }}$ approximately as the mean and standard deviation of the internal variability vary with $h_{0}$. This suggests that even time-varying forcing, with no steady component, might be able excite the internal variability of the system, in which case the direct influence of the forcing time dependence is less important.

The results of this section yield two key points: First, at low forcing amplitude, no internal variability is excited and all stratospheric variability is directly related to the forcing time dependence. Second, at high forcing amplitude, the response only sees the mean forcing amplitude and resembles the internal variability. In Part I it was found that the critical amplitude of steady forcing for which internal variability arises is $h_{0}=300$. This is consistent with Fig. 10, where it is seen that sudden warmings become increasingly decorrelated from the forcing peaks above $a_{f}=60$.

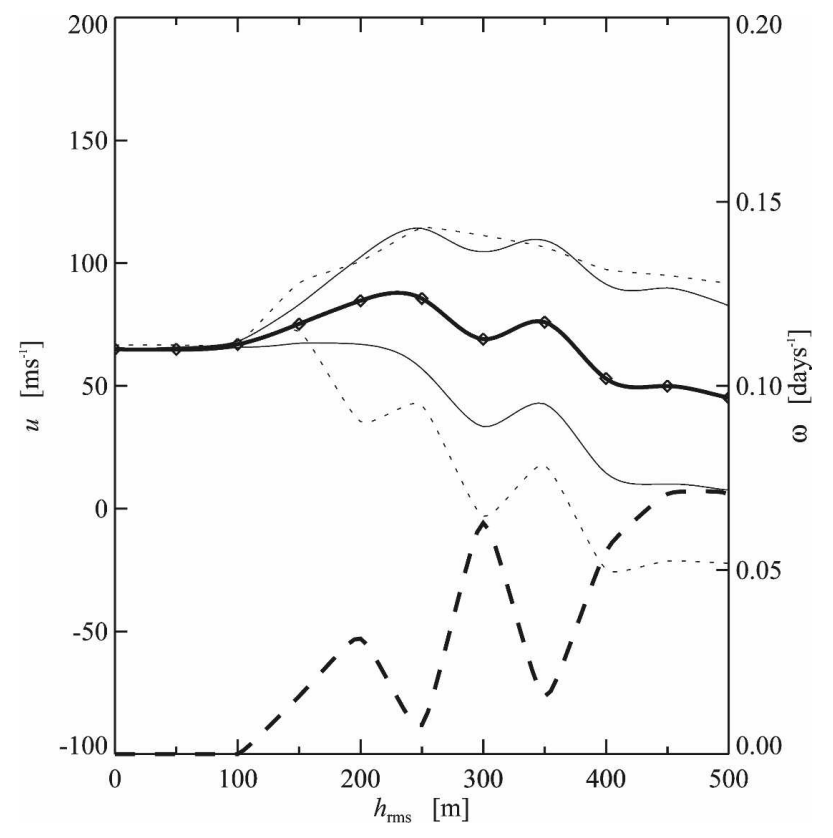

FIG. 11. Measures of $u_{j}$ (defined as $\bar{u}$ at $\phi=60^{\circ}$ and $z=41 \mathrm{~km}$ ) mean and variability as a function of rms forcing amplitude $h_{\mathrm{rms}}$ : mean (heavy solid; each diamond indicates a simulation); standard deviation (light solid, drawn relative to mean); envelope (light dotted), defined as the average maximum and minimum jet speed; and median frequency (heavy dashed), defined as the frequency for which $E(\omega)$ is maximum.

\section{Conclusions}

The main conclusion from the above results is that internal stratospheric variability, the response to purely steady forcing, is important even when the actual stratospheric forcing is time dependent. Time dependence in the forcing can modulate the internal variability, through frequency locking of the response to the forcing. However, under circumstances from which the internal variability is excited, it cannot be said that individual sudden warming events are caused directly by time-dependent pulses in the wave forcing; they are also determined by the state of the stratosphere itself. Further, the internal variability is excited not just under steady forcing conditions, but also whenever the rms forcing amplitude is above a critical threshold, even when there is no steady component to the forcing. This threshold is similar in magnitude to the threshold for internal variability in the steady forcing case.

These results shed light on why the relation between tropospheric sources and individual stratospheric sudden warming events has proven to be elusive. Attempts to correlate tropospheric quantities such as wave amplitudes with the stratospheric zonal winds generally have been unsuccessful (B. Christiansen 2006, personal 
communication). The results from section $4 \mathrm{~b}$ would suggest that any such correlation would be found only for cases when the internal variability is not excited, that is, when the rms forcing amplitude is small. This regime might correspond more closely to the Southern Hemisphere. Our experiments with periodic forcing modulation suggest that the EP flux at the lower boundary is only partially related to the forcing, but also contains a component that is directly linked to the state of the polar vortex. This is consistent both with previous model studies (Scott and Polvani 2004; Esler and Scott 2005) and with observations (Polvani and Waugh 2004). Of course, the way that waves are forced at the lower boundary in our simple model, via a specified geopotential, is different from the way waves arise in the stratosphere-troposphere system, and the lower boundary condition will clearly affect the extent to which wave propagation is modulated by the stratospheric vortex. However, we note in this regard that Scott and Polvani (2004) also found coherent variability similar to that discussed here in a model with a tropospheric wave source.

One difficulty in relating the present work to actual stratospheric variability is the lack of seasonal cycle in the model. In particular, it remains to be seen to what extent the concept of internal stratospheric variability can be applied in the case when the seasonal cycle is present. The typical period of variability obtained under perpetual winter conditions is around 60 days, which would imply one or at most two sudden warmings in a single winter, consistent with observations. Such a frequency is not inconsistent with the observed frequency of sudden warming (Charlton and Polvani 2007), and it is possible at least that internal variability is playing a role in the atmosphere.

Another artificial simplification in our model is that the topography is stationary in phase (though not steady); that is, it is composed of equal eastward- and westward-propagating traveling waves. An interesting extension would be to look at the dependence of variability on transient forcing. Esler and Scott (2005) demonstrated that the polar vortex evolution is sensitive to the phase speed of tropospheric wave forcing, depending on whether the system is able to approach conditions of resonance. An interesting question, for example, is whether transient $h$ at the resonant frequency could lead to a sudden warming via self-tuning resonance, even though $h_{\text {rms }}$ is below the critical $h$ required for internal variability. An example of this was demonstrated in Esler et al. (2006) for the case of the Southern Hemisphere 2002 major warming.

Finally, our results have implications for the study of the stratospheric response to climate change. If, despite the above caveats, the results of the simple model used here and in Part I can be considered to be partially relevant to variability of the stratosphere, then we are led to conclude that changes in the radiative conditions of the stratosphere alone will lead to changes in the stratospheric variability (see also Fig. 12 of Part I, showing the response to different $\tau_{\text {rad }}$ ). These changes in stratospheric variability would have to be considered separately from those arising from climate-induced changes in the tropospheric wave forcing; instead, they would arise directly from changes in, for example, concentrations of stratospheric water vapor or ozone. This is related to the results of Polvani and Kushner (2002), which showed that changes to stratospheric radiative conditions affected the position of the tropospheric jet. An interesting issue therefore will be to determine in more detail how internal stratospheric variability depends on radiative forcing.

Acknowledgments. Support for this research was provided by the U.S. National Science Foundation.

\section{REFERENCES}

Andrews, D. G., J. R. Holton, and C. B. Leovy, 1987: Middle Atmosphere Dynamics. Academic Press, 504 pp.

Charlton, A. J., and L. M. Polvani, 2007: A new look at stratospheric sudden warmings. Part I: Climatology and modeling benchmarks. J. Climate, 20, 449-469.

Christiansen, B., 2000: Chaos, quasiperiodicity, and interannual variability: Studies of a stratospheric vacillation model. J. Atmos. Sci., 57, 3161-3173.

Esler, J. G., and R. K. Scott, 2005: On the excitation of transient Rossby waves on the polar stratospheric vortex and the barotropic sudden warming. J. Atmos. Sci., 62, 3661-3682.

_ L. M. Polvani, and R. K. Scott, 2006: The Antarctic stratospheric sudden warming of 2002: A self-tuned resonance? Geophys. Res. Lett., 33, L12804, doi:10.1029/2006GL026034.

Holton, J. R., and C. Mass, 1976: Stratospheric vacillation cycles. J. Atmos. Sci., 33, 2218-2225.

Kushner, P. J., and L. M. Polvani, 2006: Stratosphere-troposphere coupling in a relatively simple AGCM: Impact of the seasonal cycle. J. Climate, 19, 5721-5727.

Matsuno, T., 1971: A dynamical model of the stratospheric sudden warming. J. Atmos. Sci., 28, 1479-1494.

Polvani, L. M., and R. Saravanan, 2000: The three-dimensional structure of breaking Rossby waves in the polar wintertime stratosphere. J. Atmos. Sci., 57, 3663-3685.

— spheric perturbations in a relatively simple general circulation model. Geophys. Res. Lett., 29, 1114, doi:10.1029/ 2001 GL014284.

— and D. W. Waugh, 2004: Upwelling wave activity flux as a precursor to extreme stratospheric events and subsequent 
anomalous surface weather regimes. J. Climate, 17, 35483554.

Scott, R. K., and P. H. Haynes, 2000: Internal vacillations in stratosphere-only models. J. Atmos. Sci., 57, 2333-2350.

— and - 2002: The seasonal cycle of planetary waves in the winter stratosphere. J. Atmos. Sci., 59, 803-822.

— wave flux near the tropopause. Geophys. Res. Lett., 31, L02115, doi:10.1029/2003GL017965.

— and _ 2006: Internal variability of the stratospheric polar vortex. Part I: Time-independent forcing. J. Atmos. Sci., 63, 2758-2776.

Taguchi, M., T. Yamaga, and S. Yoden, 2001: Internal variability of the troposphere-stratosphere coupled system simulated in a simple global circulation model. J. Atmos. Sci., 58, 31843203.

Waugh, D. W., and D. G. Dritschel, 1999: The dependence of Rossby wave breaking on the vertical structure of the polar vortex. J. Atmos. Sci., 56, 2359-2375.

Yoden, S., 1987: Bifurcation properties of a stratospheric vacillation model. J. Atmos. Sci., 44, 1723-1733. 